Strahlenther Onkol 2012 $\cdot 188: 461-463$ DOI 10.1007/s00066-012-0092-5

Published online: 24 March 2012

(c) Springer-Verlag 2012

M.L. Sautter-Bihl ${ }^{1} \cdot$ F. Sedlmayer ${ }^{2} \cdot$ W. Budach ${ }^{3} \cdot$ J. Dunst ${ }^{4} \cdot$ P. Feyer ${ }^{5} \cdot$ R. Fietkau ${ }^{6} \cdot$

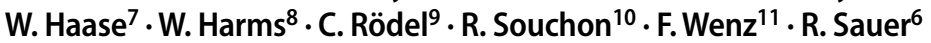

${ }^{1}$ Klinik für Radioonkologie und Strahlentherapie, Städtisches Klinikum Karlsruhe, Karlsruhe

2 University Hospital, Landeskrankenhaus Salzburg, Salzburg

${ }^{3}$ University Hospital Düsseldorf, Düsseldorf

${ }^{4}$ University Hospital Schleswig Holstein, Lübeck

${ }^{5}$ Klinikum Neukolln, Berlin

${ }^{6}$ University Hospital Erlangen, Erlangen

${ }^{7}$ Formerly St. Vincentius Kliniken, Karlsruhe

${ }^{8}$ St. Clara Hospital, Basel

${ }^{9}$ University Hospital Frankfurt/Main, Frankfurt/Main

${ }^{10}$ University Hospital Tübingen, Tübingen

${ }^{11}$ University Medical Center Mannheim, Mannheim

\title{
One life saved by four prevented recurrences?
}

\author{
Update of the Early Breast Cancer \\ Trialists confirms: postoperative \\ radiotherapy improves survival \\ after breast conserving surgery
}

ly awaited the quinquennial update of the EBCTCG which has now been published [6].

Patients and methods. A total of 17 randomized studies comparing postoperative radiotherapy vs. none were analyzed and comprised 7 new studies in addition to follow-up data of from 9 previously reported trials. A total of 10,801 patients with pT1-2 tumors were included, the majority of whom $(n=7,287)$ were node negative, while 1,050 were node positive (2,464 unknown). In contrast to the previous meta-analysis [5], all patients received breast conserving surgery, consisting of lumpectomy $(n=8,422)$ or more extensive techniques like quadrantectomy or sectoral resection $(n=2,399)$.

The effect of radiotherapy on 10-year recurrences of any type and their relation to the 15-year breast cancer death rate were studied in correlation to various prognostic parameters and treat- ment characteristics (e.g., surgery, tamoxifen use). Moreover, a subgroup analysis was performed according to low, intermediate, and high initial risk of recurrence, from which the expected absolute benefit was derived by respective modeling of the prognostic factors.

Results. Overall, radiotherapy reduced the 10-year recurrence rate (local or distant) from $35 \%$ to $19.3 \%$, corresponding to an absolute benefit of $15.7 \%(2 p<0.0001)$ for irradiated women (node negative (pN0) $15.4 \%$, node positive ( $\mathrm{pN}+$ ) $21.2 \%$ ). The 10-year rate of locoregional recurrence as first event was substantially higher for non-irradiated women: 25.1 vs. $7.7 \%$, i.e., an absolute difference of $17.4 \%$ (pN0 15.5\%, pN+30.6\%) ([6] web Fig. 2 $\mathrm{a}, \mathrm{b}, \mathrm{c})$.

Moreover, radiotherapy reduced the 15 -year breast cancer death rate from $25.2 \%$ to $21.4 \%$ corresponding to an absolute gain of $3.8 \%$ (pN0 3.3\%, pN+ 8.5\%).

[5]. The scientific community has eager- 


\begin{tabular}{|c|c|c|c|c|c|c|}
\hline & \multicolumn{2}{|c|}{ All patients $(n=10,801)$} & \multicolumn{2}{|c|}{ pN0 $(n=7,287)$} & \multicolumn{2}{|c|}{$\mathrm{pN}+(\mathrm{n}=1,050)$} \\
\hline & BCS & $\mathrm{BCS}+\mathrm{RT}$ & BCS & $\mathrm{BCS}+\mathrm{RT}$ & BCS & $\mathrm{BCS}+\mathrm{RT}$ \\
\hline $\begin{array}{l}\text { 10-year any } \\
\text { recurrence } \\
\text { (\%) }\end{array}$ & 35.0 & 19.3 & 31.0 & 15.6 & 63.7 & 42.5 \\
\hline $\begin{array}{l}\text { 10-year lo- } \\
\text { coregional } \\
\text { first (\%) }\end{array}$ & 25.1 & 7.7 & 22.8 & 7.3 & 43.0 & 12.4 \\
\hline $\begin{array}{l}\text { 10-year } \\
\text { distant first } \\
\text { (\%) }\end{array}$ & 9.9 & 11.6 & 8.2 & 8.3 & 20.7 & 30.1 \\
\hline $\begin{array}{l}\text { 15-year BC } \\
\text { mortality } \\
(\%)\end{array}$ & 25.2 & 21.4 & 20.5 & 17.2 & 51.3 & 42.8 \\
\hline
\end{tabular}

Tab. 2 Comparison of the 15-year breast cancer mortality in the EBCTCG meta-analyses 2005 [5] and 2011 [6]

\begin{tabular}{|lllll}
\hline & 2011 & & 2005 & \\
\hline & $+\mathbf{R T}$ & $-\mathbf{R T}$ & $+\mathbf{R T}$ & $-\mathbf{R T}$ \\
\hline $\mathrm{pN} 0$ & 17.2 & 20.5 & 26.1 & 31.2 \\
\hline $\mathrm{pN}+$ & 42.8 & 51.3 & 47.9 & 55.0 \\
\hline RT radiotherapy, $\mathrm{pN0}$ node negative, $\mathrm{pN}+$ node positive. & & \\
\hline
\end{tabular}

Finally, radiotherapy reduced the 15 year risk of any death from $37.6 \%$ to $34.6 \%$, providing an absolute gain of $3.0 \%$ (pN0 $2.8 \%, \mathrm{pN}+10.7 \%)$. Mortality without recurrence was slightly but not significantly higher in irradiated women (relative risk $1.09,0.97-1.22,2 \mathrm{p}=0.14$ ).

Conclusion of the authors. Radiotherapy halved the average annual rate of disease recurrence (RR 0.52, 0.48-0.56) and reduced the annual breast cancer death rate by about one sixth (RR $0.82,0.75-0.90$ ). On average, in all patients, about one breast cancer death was avoided by year 15 for every four recurrences avoided by year 10. Little variation of the proportional benefit was seen in the different prognostic subgroups or with different types of surgery. In contrast, the absolute benefit from radiotherapy substantially depended on the patient's characteristics in terms of prognostic factors [6].

\section{Comment}

The baseline message of the previous meta-analysis [5] is once more confirmed: Local control translates into improved breast cancer specific and overall survival and even the "one-in-four relationship" is roughly corroborated. The presentation of the current study differs from the previous one as the 5-year local recurrence rate as the primary focus of analysis has been replaced by presenting 10-year "first" recurrence of any type, which have been halved by radiotherapy. In his comment on these data, T.A. Buchholz [2] stated: "to put this benefit into context, a $50 \%$ proportional reduction in 10-year recurrence exceeds that from chemotherapy alone or hormonal therapy alone" [2].

As radiotherapy reduces the risk of breast cancer death, eradication of residual disease obviously does not only prevent local recurrence but impedes the emergence of distant disease to a similar extent. The observation that in patients with positive axillary nodes the rate of distant disease diagnosed as first recurrence is higher after irradiation than after breast conserving surgery (BCS) alone (• Tab. 1) must not be misinterpreted: the fact that radiotherapy (RT) substantially prevents or delays local recurrence may lead to an increased proportion (but not absolute number) of patients presenting with metastases as first recurrence that would otherwise have been preceded by locoregion- al relapse. On the other hand, it is obvious that increased local control can only translate into improved survival in the absence of pre-existing distant micrometastatic disease which is not eradicated by systemic treatment [2]. Noteworthy, RT provided the largest absolute survival advantage for those women with node- positive disease $(\mathrm{pN}+8.5 \%)$. This observation suggests that even though positive axillary lymph nodes strongly indicate the potential of later distant recurrence, a fraction of metastases is not present prior to treatment but develops later on the basis of residual local disease.

This affirms the hypothesis of a "late wave of metastases" that has been propagated for prostate cancer [3] but not yet corroborated with the same extent of evidence as for breast irradiation.

In the current meta-analysis, six studies $(n=2,911)$ were confined to low risk patients with lumpectomy and adjuvant tamoxifen. Treatment results for this patient group support the assumption that the expectable absolute benefit of radiotherapy is related to the primary risk of recurrence and to the extent to which recurrence is preventable. As the authors conclude: halving a big risk provides more absolute gain than halving a small risk. While the proportional reduction of the 10 -year recurrence rate was $65 \%$ (RR 0.35 , $0.27-0.45)$ in these low-risk patients, the absolute reduction was only $13.6 \%$. This is plausible as the 10 -year recurrence rate was only $19.9 \%$ in patients without RT and $6.3 \%$ in irradiated women. For comparison: in the older lumpectomy trials with mixed risk groups, the absolute gain was $20.1 \%$ according to the fact that the 10-year recurrence rate in this group was much higher ( $47.9 \%$ without RT). Nonetheless, the proportional reduction was smaller (RR 0.53, 0.46-0.61)([6], web Fig. 5e). Interestingly, the $50 \%$ proportional reduction also applied for those patients who had more extensive surgery ([6], web Fig. 5d).

The EBCTCG update makes another important contribution to the ever-lasting discussion about radiation toxicity that may counteract the benefit of RT. Mortality without recurrence was slightly higher in the irradiated group $(1.09,2 p 0.14)$ but the excess was not significant and identi- 
cal to the excess- mortality observed by the EBCTCG for 1 year of tamoxifen vs. none [7]. The authors did not provide a further cause-specific analysis of nonbreast cancer-related deaths. Assuming that some of these might be attributable to radiation, there is definitely a high potential to further eliminate or at least reduce these effects with modern treatment techniques [1][11][12][15].

In contrast to the 2005 meta-analyses, survival has improved: the 15 -year breast cancer death rate was lower in the present study (• Tab. 2; [5][6]. Patient groups may not be totally comparable because of the new studies with different inclusion criteria and adjuvant systemic treatment. Nonetheless, this trend indicates that the tools to combat breast cancer have gained potency. The present analysis provides convincing evidence that preventing local recurrence in the first decade yields the promise of improved survival in the second. Hence, local and systemic treatment should be regarded as a corporate interdisciplinary challenge as the strength of each enforces the potential of the other.

Several new treatment modalities are currently being discussed like partial breast irradiation [10], or omitting axillary dissection (without irradiating the axilla instead!) in patients with a positive sentinel node and a clinically negative axilla [9]. This should be scrutinized with regard to the large absolute gain of $20 \%$ (proportional reduction of 50\%) in of the 10-year recurrence risk in patients with $1-3$ positive axillary nodes $(n=718$; [6], web Fig. 11a). These patients had not received nodal irradiation but as level III is supposedly covered by whole breast RT, occult disease in the axilla may "inadvertently" have received a tumoricidal dose. These findings strongly support the suggestion of Haffty et al. [9] concerning the implications of a positive SNB for the radiation oncologist: his approach is using dedicated high tangents with deliberate inclusion of level I-II in these patients.

\section{Conclusion}

Thus, it should be borne mind that in light of the large number of affected women, even small incremental changes will impact thousands of lives.

\section{Corresponding address}

Prof. Dr. M.L. Sautter-Bihl

Klinik für Radioonkologie und Strahlentherapie, Städtisches Klinikum Karlsruhe

Moltkestr. 90, 76133 Karlsruhe

Germany

marie-luise.sautter-bihl@klinikum-karlsruhe.de

\section{References}

1. Boda-Heggemann J, Lohr F, Wenz F et al (2011) kV cone-beam CT-based IGRT: a clinical review. Strahlenther Onkol 187:284-291

2. Buchholz TA (2011) Radiotherapy and survival in breast cancer. Lancet 378(9804):1680-1682

3. Coen JJ, Zietman AL, Thakral H, Shipley WU (2002) Radical radiation for localized prostate cancer: local persistence of disease results in a late wave of metastases. J Clin Oncol 20:31993205

4. Early Breast Cancer Trialists' Collaborative Group (2000) Favourable and unfavourable effects on long term survival of radiotherapy for early breast cancer: an overview of the randomized trials. Lancet 355:1757-1770

5. Early Breast Cancer Trialists' Collaborative Group (2005) Effects of radiotherapy and of differences in the extent of surgery for early breast cancer on local recurrence and 15-year survival: an overview of the randomised trials. Lancet 366:20872106

6. Early Breast Cancer Trialists' Collaborative Group (EBCTCG), Darby S, McGale P, Correa C et al (2011) Effect of radiotherapy after breast-conserving surgery on 10-year recurrence and 15-year breast cancer death: meta-analysis of individual patient data for 10,801 women in 17 randomised trials. Lancet 378:1707-1716. EBCTGC supplementary web tables DOI:10.1016/S0140-6736(11)61629-2

7. Early Breast Cancer Tiralists' Collaborative Group (EBCTCG) (2011) Relevance of breast cancer hormone receptors and other factors to the efficacy of adjuvant tamoxifen: patient-level meta-analysis of randomised trials. Lancet 378:771-784. EBCTGC supplementary web tables DOI:10.1016/ S0140-6736(11)60993-8

8. Fisher B, Anderson SJ (2010) The breast cancer alternative hypothesis: is there evidence to justify replacing it? J Clin Oncol 28:366-374

9. Haffty BG, Hunt KK, Harris JR, Buchholz TA (2011) Positive sentinel nodes without axillary dissection: Implications for the radiation oncologist. J Clin Oncol 29:4479-4481

10. Sautter-Bihl ML, Sedlmayer F, Budach W et al (2010) Intraoperative radiotherapy as accelerated partial breast irradiation for early breast cancer: beware of one-stop shops? Strahlenther Onkol 186:651-657
11. Schubert LK, Gondi V, Sengbusch E et al (2011) Dosimetric comparison of left-sided whole breast irradiation with 3DCRT, forward-planned IMRT, inverse-planned IMRT, helical tomotherapy, and topotherapy. Radiother Oncol 100:241-246

12. Tromm E, Meyer A, Frühauf J, Bremer M (2010) Partial-volume segmentation for dose optimization in whole-breast radiotherapy: a comparative dosimetric and clinical analysis. Strahlenther Onkol 186:40-45

13. Van de Steene J, Soete G, Storme G (2000) Adjuvant radiotherapy for breast cancer significantly improves overall survival: the missing link. Radiother Oncol 55:263-272

14. Vinh-Hung V, Verschraegen C (2004) Breast-conserving surgery with or without radiotherapy: pooled analysis for risks of ipsilateral breast tumor recurrence and mortality. J Natl Cancer Inst 96:115-221

15. Zurl B, Stranzl H, Winkler P, Kapp KS (2010) Quantitative assessment of irradiated lung volume and lung mass in breast cancer patients treated with tangential fields in combination with deep inspiration. Strahlenther Onkol 186:157-162 